\title{
Entre petits plaisirs et grande jouissance. Les rituels (in)avoués du lecteur ${ }^{1}$
}

José Domingues de Almeida*

Université de Porto - ILCML

Résumé : Nous passerons en revue plusieurs considérations dégagées dans plusieurs essais critiques récents sur l'acte de lire pris dans ses rituels et ses apories diverses. C'est toujours la question du plaisir de la lecture qui est abordées et interrogée à partir de l'expérience de la vie quotidienne.

Mots-clés : lecture, plaisir, rituels, livre

Abstract: We will review several considerations identified in several recent critical essays on the act of reading taken in its various rituals and apories. It is always the question of the pleasure of reading that is addressed and questioned from the experience of daily life.

Keywords: reading, pleasure, rituals, book

Un article rafraîchissant signé Nicolas Ancion dans un numéro thématique d'Écritures consacré au concept de postmodernité appliqué au fait littéraire avait attiré l'attention en 1993 de plus d'un critique sur l'autre face (lectrice) de l'exercice littéraire. Ancion fondait sa caractérisation du lecteur contemporain sur les apports théoriques de Roland Barthes dans S/Z (1970) et surtout dans Le Plaisir du texte (1973). L'article convoquait le concept opératoire de "protocole de lecture » par lequel le lecteur assigne une fonction au texte en raison du « but qu'[il] confère à sa lecture, et qui ressortit aussi bien à la nature du texte qu'à la compétence lectrice » 
(Ancion 1993 : 30-31), et dont l'infinitude implique le regroupement en « modalités protocolaires » (idem : 32 ).

Trois, en particulier, s'en dégagent qui ont respectivement trait à la lecture de plaisir, à savoir la reconnaissance et la recréation du sens préexistant et indéfini du texte - une modalité que nous pratiquons tous à l'envi comme chercheurs en littérature -, à la lecture de devoir en vertu de la scolarité obligatoire, et finalement à la lecture de jouissance, laquelle se replie de façon jubilatoire sur l'acte de lecture en soi plutôt que sur le texte lu, cette modalité visant un discours neuf, non encore exprimé (Marcoin 1990 : 133-145).

Rappelons que Roland Barthes voit dans le texte de plaisir un «parler sur » (1973 : 37), où le langage se superpose à la parole, ce qui rend ce plaisir dicible (idem : 8283), tandis que le texte de jouissance s'avérerait « intenable », « impossible », ce qui l'associe à un « parler en » (idem: 37), « hors de toute finalité imaginable » (idem: 83). Là, forcément, on entre dans le domaine de l'atopie du texte, en situation « hors-critique » (idem : 37).

Ancion met l'hypothèse selon laquelle l'épuisement des ressources textuelles modernes à engendrer du plaisir critique (au sens barthésien) peut mobiliser chez le lecteur une créativité et une liberté qui le poussent à s'exprimer sur ses rituels dans la pratique de la lecture littéraire. Ceci suppose bien évidemment primo : que le livre se porte bien, spécialement en bibliothèque, ce que révèlent de récentes statistiques concernant le prêt, notamment numérique, comme c'est le cas en Wallonie-Bruxelles, ${ }^{2}$ et secundo: que le livre en format papier a toujours la cote. À cet égard, une étude assez récente faisait état des réticences des Français à lire le texte littéraire sur tablette ou liseuse et ce, pour deux raisons majeures : l'écart de prix insuffisant entre les formats papier et numérique, ${ }^{3}$ et l'attachement affectif au livre en version papier (Habrand $2009: 11$ ), ce produit symbolique indéboulonnable dans la « nation littéraire » par excellence qu'est la France (Ferguson 1991). Ce cadre a été encore dernièrement rappelé par Pierre Schoentjes, citant Danièle Sallenave, pour qui la lecture des grands textes littéraires par la jeunesse immigrée en mal d'intégration, notamment en France et en Belgique - deux pays simultanément foyers et victimes du terrorisme islamiste - permettrait une meilleure insertion dans le roman national et dans l'adhésion aux idéaux de tolérance et de laïcité (Schoentjes 2019).

La mise en fiction de cet idéal de promotion personnelle et d'intégration culturelle par la pratique lettrée et la scolarisation en général devient même un véritable topos des littératures dites (post)-migrantes (Albert 2005 ; Declercq 20II, et Delbart 20IO), où bien souvent le personnage entend douloureusement s'émanciper des entraves de la culture d'origine et familiale en se réfugiant dans la pratique lectrice, et notamment des grands auteurs (Begag 1986 ; Chahdortt 2006 ; Khadra 2008 ; Laroui 2011; Madi 2013, et Madjidi 2017).

Mais revenons sur ces rituels et fantasmes de lecture que la modalité jubilatoire 
rend plus facilement avouables quand les langues de lecteur se délient. Le succès international de Comme un roman de Daniel Pennac (1992) a révélé l'aspiration du lectorat à concevoir la pratique de la lecture littéraire comme une activité foncièrement libre et jouissive, ainsi que sa désaffection par rapport aux modalités du plaisir critique et du devoir scolaire. Rappelons d'entrée de jeu l'aphorisme de Pennac: «le verbe lire ne supporte pas l'impératif » (Pennac 1992: 13), ainsi que sa définition de la lecture comme "acte de création permanente » (idem : 27). Aussi l'auteur de Chagrin d'école (2007) substitue-t-il à la logique du devoir celle de "droits" du lecteur: ne pas (vouloir) lire, sauter des pages ou limiter sa lecture à la quatrième de couverture, commencer une lecture et ne pas la finir, relire, lire n'importe quoi, s'identifier aux personnages, lire n'importe où, passer d'un livre à l'autre, lire à haute voix ou se taire (Pennac 1992: 165-198).

Le droit à la non-lecture, ou les ruses pour la camoufler socialement, particulièrement dans la production sociale du « discours tenu sur les livres » a fait l'objet de Comment parler des livres qu'on n'a pas lus? de Pierre Bayard (Bayard 2007 : 14). Il dégage une des conditions de la pratique lectrice innommée chez Pennac : la «non-lecture », voire la « délecture ». Celles-ci éclairent l'entre-deux de la lecture étant donné le discours attendu socialement, voire académiquement, sur le fait littéraire, la réalisation de cette épreuve engageant un éventail de stratégies, par exemple la mobilisation d'effets de réel savamment placés pour faire accroire qu'on a lu : "livre inconnu », «livre évoqué », "livre oublié », "livre parcouru ». Plus récemment, Maxime Decout faisait l'Éloge du mauvais lecteur (202I) où il signalait l'art, de l'adresse et la ruse qu'il y a à pratiquer une mauvaise lecture, une lecture qui ne cherche pas l'objectivité de l'interprétation, mais se perd dans la maladresse lectrice assumée. Dans le même sens, Daniel Simon, sur l'essai duquel nous reviendrons plus tard, évoquait ce sentiment d'angoisse et de dépassement éprouvé par le lecteur en librairie: « (...) toutes ces œuvres qu'on ne lira pas. Ces chefs-d'œuvre qui le resteront sans nous » (Simon 2019:30), voire ces livres qu'on croit avoir lu (idem : 42).

Il y aurait donc une sorte d'indicibilité de l'expérience lectrice placée dans l'entre-deux sujet-discours, et qui fait apparaître un "livre intérieur ", "transmissible à personne et superposable à aucun autre (...) » (idem : 94), subjectif, mais aux implications plus profondes qu'il n'y paraît au premier abord. Le "livre-fantôme " (idem : 140) dont parle Pierre Bayard dessine, tout compte fait, des fantasmes et des non-dits autour de la lecture littéraire, qui la font apparaître, selon la formule de Valery Larbaud, comme "ce vice impuni ».

Or, cette passion avait fait l'objet d'un livre-aveux de bibliophage ${ }^{4}$ de la part de l'éditrice au Seuil, la regrettée Annie François, intitulé Bouquiner. Autobiobibliographie (2000) dans lequel la « liseuse » insatiable et incurable s'inscrivait, non sans humour, dans la foulée d'un Pennac. Le lecteur y est vu comme « un asocial, solitaire, une sorte d'autiste. Essayez de l'empêcher de finir son paragraphe. L'être le plus amène 
s'ensauvage. Tant qu'il n'a pas reposé son livre de plein gré, c'est un être potentiellement dangereux " (François 2000 : 74). François y passe en revue les interactions de la pratique de la lecture avec les sens, c'est-à-dire la vibrante matérialité de l'acte de lecture, la lecture convoquant le corps. Et tout d'abord, la position adoptée pour lire : «Depuis toujours, pour moi, livre et lit sont associés » (idem : 9), ce que l'éditrice reformule comme suit : «Bref, je ne lis bien qu'au lit, ou plutôt allongée. Jadis sur le ventre, aujourd'hui sur le dos, solidement calée par deux oreillers. La lecture assise reste associée à l'école, au travail, à la contrainte par corps » (idem: II).

D’ailleurs, Philippe Delerm ne se plaignait-il pas dans La première gorgée de bière et autres plaisirs minuscules de l'inconfort causé, ou du défi relevé par la lecture sur la plage:

À lire trop longtemps les bras étalés devant soi, le menton s'enfonce, la bouche boit la plage, alors on se redresse, bras croisés contre la poitrine, une seule main glissée à intervalle pour tourner les pages et les marquer. C'est une position adolescente, pourquoi? Elle tire la lecture vers une ampleur un rien mélancolique. Toutes ces positions successives, ces essais, ces lassitudes, ces voluptés irrégulières, c'est la lecture sur la plage. On a la sensation de lire avec le corps. (Delerm 1997 : 47)

Et tout y est récapitulé sous forme de rituels liés aux petits plaisirs de la lecture trahissant en fait une véritable jouissance à laquelle tous lecteurs enthousiastes que nous sommes s’identifieront, et qui exclut définitivement le livre de la logique marchande: «Or, le livre à mes yeux ne saurait être une marchandise. J'écume de la voir affublé de cette herse [le code-barres] qui plombe les dos de couverture, affiche le triomphe des gestionnaires sans concession à l'esthétique » (idem : 60).

C’est le cas de la stratégie de marquage des pages : "Sans être fétichiste, je ne saurais corner les pages. Je ne peux pourtant me résoudre à adopter le signet » (idem : 12), tandis que « (...) gribouiller sur l'ultime page blanche me plaît » (idem : 68). La répugnance du marque-page fait en sorte que " [ses] livres sont fourrés d'articles, de vieilles lettres, de listes de courses » (idem : 14), ou encore de la panique causée par le prêt de ses livres : "Le prêt spontané est encore plus accablant. Masochiste, on est l'artisan de son propre malheur. Avec, en prime, l'auréole de la générosité ou le remords de la suffisance (...) » (idem: 16). Annie François, qui rechigne à la fréquentation de la bibliothèque publique tout en reconnaissant qu'elle permet au moins une dynamique de circulation pour le livre (idem : 25), et qui se dit attentive aux odeurs variables exhalées par les livres ouverts (idem : 47-50), s'attarde sur le grand détail de la couverture: "J'appartiens à cette génération de lecteurs pour qui le passage à l'âge préadulte était signifié par un brutal basculement de la couverture illustrée à l'austère couverture typo » (idem : 43).

Mais d'autres fantasmes et phobies de lecteur sont révélés au grand jour : les 
difficultés et dilemmes du rangement (idem : ॥O-॥4), l'irritation causée par la curiosité d'autrui : "Ainsi, je ne supporte pas qu'on regarde le titre de mon livre, surtout quand l'œil de l'indiscret exprime cette pénible certitude ‘Dis-moi ce que tu lis...' (...) » (idem: 119), "Je ne supporte pas non plus qu'on lise par-dessus mon épaule. J'ai l'impression qu'on entre dans mon bain » (idem: 121). Les questions pratiques liées aux déplacements ne sont pas non plus éludées. Quels livres prend-t-on avec soi, et quels autres sacrifie-t-on? Comment les dispose-t-on une fois logés dans une chambre d'hôtel que l'on partage avec quelqu'un ?: " Le choix final dépend du mode de transport, de la longueur du séjour, de la destination, des participants. J'ai d'ailleurs appris que certain grand navigateur n'autorise à ses équipiers que 172 pages maximum » (François $2000: 124$ ).

Mais si la lecture est avant tout source de plaisir, elle est collatéralement aussi cause de douleurs, notamment «(...) l'arthrose cervicale qui guette tout lecteur penché sur son ouvrage » (idem: II5), et les troubles du sommeil et bipolaires: «La lecture rend insomniaque. Le lecteur rate délibérément le 'le train du sommeil' (...). Le lecteur est capable de se crever les yeux à la lumière mourante d'une lampe de poche, d'un réverbère, d'un néon clignotant, d'une veilleuse de voiture, d'une bougie » (idem:॥6). La lecture en vient même à avoir la part belle par rapport au sexe, elle revendique sa priorité : «Et même dans le couple, à l'heure des petits câlins ('Juste une minute, je termine mon chapitre'). Une demi-heure plus tard, l'autre dort» (idem : 168). À cet égard, un passage du livre culte de Jean-Philippe Toussaint, La salle de bain, est saisissant. Le narrateur est surpris pendant sa lecture par la pressante envie de son amoureuse, une certaine Edmondsson, de faire l'amour. Il hésite entre ces deux jouissances rivales, et finit par s'exécuter, non sans d'abord mettre son autre « vice » en suspens, voire en souffrance :

(...) I3) Assis sur mon lit, le dos contre un oreiller, je lisais. La porte d'entrée claqua, je relevai la tête. Un instant plus tard, Edmondsson apparaissait, le visage rayonnant. Elle voulait faire l'amour. 14) Maintenant. 15) Faire l'amour maintenant? Je refermai mon livre posément, laissant un doigt entre deux feuilles pour me garder la page. (Toussaint 2005 [1985]: 17)

Ceci nous amène à considérer une ergonomie - souvent proche, du reste - d'une érotique de la lecture, qui prend sa source dans la tendre enfance. Annie François conseille vivement aux adultes de lire Comme un roman de Pennac (idem : 165), pour surtout ne pas obliger les enfants à lire, la jouissance de la lecture advenant essentiellement de la «transgression» (ibidem).

Un récent essai de l'animateur d'atelier d'écriture, dramaturge et poète belge, Daniel Simon, revient sur ces questions dans Positions pour la lecture (2019), salué pour son « didactisme » ${ }^{5}$. Bien évidemment " positions » (au pluriel) est à prendre 
dans sa polysémie (prise de position et posture physique, ouverture aux sens). À nouveau, l'enfance est pointée comme la source du désir ultérieur de lire : "Ce sont les lectures de l'enfance qui creusent le puits. Après, ça 'coule de source' » (Simon 2019: 7). Et Simon d'apparenter subtilement ce plaisir «solitaire" (idem:15) à la masturbation, et aux interdits sociaux qui la proscrivent.

Daniel Simon souligne d'entrée de jeu le caractère sensoriel, érotique et égotiste de la pratique de la lecture : "Pour être dans la lecture, il s'agit d'être en retrait du réel » (idem : 8), puisque «le lecteur lit dans un corps qui lui échappe, qui glisse vers l'absence momentanée »(ibidem). Ce sentiment est éprouvé notamment quand quelqu'un lit, acte solitaire, au milieu de la foule, en contexte social comme, par exemple, dans un transport public bondé : "Lire alors, se soustraire, disparaître souverainement ( (idem : IO). La lecture solitaire et silencieuse engage aussi implicitement l'ouïe, une voix qui cherche à se rendre audible : « (...) on murmure un texte pour soi, parfois, tellement c'est beau et qu'on voudrait aussi l'entendre de l'extérieur de soi » (idem : 16).

En animateur d'atelier d'écriture, Simon se place volontiers du côté du lecteur, qu'il est lui-même, et qui l'a conduit à l'écriture : "J'ai l'habitude de lire de diverses façons. Un livre, une position de lecture... Entre-temps, je feuillette, je picore, je bouquine » (idem : 22). «Bouquiner », un verbe qu'Annie François avait retenu pour son titre, et qui renvoie aussi bien à la quête qu'à la dévoration quasi matérielle d'un livre. Et cette faim convoque le corps et sa posture. Tout comme François, Simon avoue préférer la position couchée pour la lecture, comme on choisit une position pour faire l'amour, un kamasoutra de lecteur : « II avait depuis longtemps choisi la position couchée lors de ses activités de lecture (...), c’était dans ce mol repli que tout s'ouvrait (...)» (idem : 29).

Bien évidemment, cette approche de l'acte jouissif de la lecture l'inscrit dans un contexte historique, amplement décrit par Alfredo Manguel (1998), de lecture intime et privée, qui s'imposera, laquelle justement dégage l'activité lectrice comme plaisir solitaire, mais aussi subliminale projection du lecteur dans une fiction et qui permet à de l'inavoué d'affranchir ses propres fantasmes de la censure sociale (idem : 304). En effet, si la pratique liseuse littéraire mobilise toujours, certes, des compétences acquises à l'école (Falardeau \& Sauvaire 2015 : 71-84), convoque toujours majoritairement la version imprimée et s'inscrit toujours quelque part dans un certain dessein social, elle « (...) a rompu son lien essentiel au 'Livre', pierre d'angle d'une civilisation humaniste, née de la république des Lumières, désormais anéantie " (Chartier \& Hébrard 2000). Elle revendique son plaisir intrinsèque, lequel se diffracte dans plusieurs rituels éprouvés, souvent inavoués, mais dont on lit la description personnelle dans les deux essais post-Comme un roman évoqués ici. Celui-ci culmine dans la lecture les "yeux fermés »: "Le texte, les personnages, tout est là devant eux, à un moment ça suffit, on ferme les yeux et le texte continue sur sa lancée intérieure (...) 
» (idem : 45) car, en effet, " la lecture a toujours un effet retard, comme ces étoiles mortes dont la lumière nous parvient en continu... » (ibidem).

\section{Notes}

* José Domingues de Almeida est Maître de Conférences à la Faculté des Lettres de l'Université de Porto. Il est docteur en littérature francophone contemporaine. Ses domaines de recherche sont la littérature française contemporaine, les études francophones et la culture et pensée françaises contemporaines. Il se penche récemment sur les questions théoriques et critiques soulevées par les littératures post-migratoires, les récits post-mémoriels et les représentations de l'Europe. Il est chercheur à l'Instituto de Literatura Comparada Margarida Losa et directeur de la revue électronique Intercâmbio. Il est, par ailleurs, vice-président de l’Association Portugaise d’Études Françaises.

' Cet article est developé dans le cadre de l'Institut de Littérature Comparé, Unité R\&D financié par fonds nationaux de la FCT - Fondation pour la science et la technologie (UIDP/00500/2020).

${ }^{2}$ Voir https://le-carnet-et-les-instants.net/2020/0I/20/chiffres-des-bibliotheques/ .

${ }^{3}$ On lira avec profit l'étude de Jean Clément sur la lecture et le numérique qui va bien plus loin que les enjeux purement ergonomiques, notamment pour ce qui est de l'“hyperlecture” (2001 : I13-134). Voir aussi, pour l'approche de ces mutations, le chapitre « Pratiques de lecture et livres électroniques » (van Cuyck \& Bélisle 2004 : 77-IOI).

${ }^{4}$ À l'opposé, la neurasthénie du lecteur s'avère un mauvais symptôme : « Pour un lecteur, même modeste, le désamour de la lecture constitue un symptôme. 'Je n'ai même plus envie de lire' signifie qu'on a atteint le fond de la dépression, de la fatigue, du chagrin » (François 2000 : IOI). D’ailleurs, « Si un livre n’arrive pas à me tenir après trente pages, je l'évacue. Il végète alors auprès du lit, attendant des jours meilleurs, une autre saison, un état de grâce, des circonstances favorables, un intercesseur brillant » (idem : 184).

${ }^{5} \mathrm{https} / / /$ le-carnet-et-les-instants.net/2019/10/23/simon-positions-pour-la-lecture/ . 


\section{Bibliographie}

Albert, Christiane (2005), L'Immigration dans le roman francophone contemporain, Paris, Karthala.

Ancion, Nicolas (1993), "La lecture littéraire, entre post- et modernité », in Écritures, nO 5, «Le dépli. Littérature et postmodernité », pp. 30-38.

Bayard, Pierre (2007), Comment parler des livres qu'on n'a pas lus ?, Paris, Minuit. Begag, Azouz (1986), Le Gone du Chaâba, Paris, Seuil.

Chahdortt, Djavann (2006), Comment peut-on être français ?, Paris, Flammarion.

Clément, Jean (200I), «La littérature au risque du numérique »,Document numérique, vol. 5, pp. 113-134.

Cuyck, Alain van et Bélisle, Claire (2004), "Pratiques de lecture et livres électroniques », in $\mathrm{Cl}$. Bélisle (Éd.), La lecture numérique: réalités, enjeux et perspectives. Villeurbanne: Presses de l’enssib, coll. «Référence », pp. 77-ı।I.

Declercq, Elien (20II), «Écriture migrante: réflexions sur un concept aux contours imprécis ", Revue de Littérature Comparée, no 339, pp. 301-310.

Decout, Maxime (2021), Éloge du mauvais lecteur, Paris, Minuit.

Delbart, Anne-Rosine (2010), "Littératures de l'immigration : un pas vers l'interculturalité? », Carnets («Littératures nationales: suite ou fin - résistances, mutations \& lignes de fuite »), no spécial printemps/été, pp. 99-110.

Delerm, Philippe (1997), La première gorgée de bière et autres plaisirs minuscules, Paris, L’Arpenteur.

Falardeau, Érick et Sauvaire, Marion (2015), «Les composantes de la compétence en lecture littéraire ", Le Français aujourd'hui, Paris, Armand Colin, nO 191, pp. 71-84.

Ferguson, Priscilla Parkhurst (1991), La France, nation littéraire, Bruxelles, Labor.

François, Annie (2000), Bouquiner. Autobiobibliographie, Paris, Seuil.

Habrand, Tanguy (2009), "L'édition à l'heure du numérique », Le Carnet et les Instants, no 156, pp. 2-II.

Khadra, Yasmina (2008), Ce que le jour doit à la nuit, Paris, Julliard.

Laroui, Fouad (2010), Une année chez les Français, Paris, Julliard.

Madi, Malika (2013), Nuit d'encre pour Farah, Mons : Éd. du Cerisier.

Madjidi, Maryam (2018), Marx et la poupée. Paris, Le Nouvel Attila.

Manguel, Alberto (1998), Uma história da leitura, Lisboa, Editorial Presença.

Marcoin, Francis (1990), «La lecture littéraire: une didactique de l'invisible », Spirale. Revue de recherches en éducation, $\mathrm{n}^{\circ}$ 3, pp. 133-145.

Pennac, Daniel (1992), Comme un roman, Paris, Gallimard.

Simon, Daniel (2019), Positions pour la lecture, Mons, Couleurs livres, coll. « Je Contrepoints ».

Toussaint, Jean-Philippe (2005), [1985]. La salle de bain, Paris, Minuit. 


\section{Sitographie:}

https://le-carnet-et-les-instants.net/2019/10/23/simon-positions-pour-la-lecture/ https://le-carnet-et-les-instants.net/2020/0I/20/chiffres-des-bibliotheques/ 